\title{
Review Article \\ Role of Noncoding RNA in Development of Nonalcoholic Fatty Liver Disease
}

\author{
Ruixian Huang $(\mathbb{D}$, Xiaoyan Duan, Jangao Fan $\mathbb{D}$, Guangming Li $\mathbb{D}$, and Baocan Wang $\mathbb{D}$ \\ Center for Fatty Liver, Department of Gastroenterology, Xin Hua Hospital affiliated to Shanghai Jiao Tong University School of \\ Medicine, Shanghai 200092, China \\ Correspondence should be addressed to Guangming Li; ligm68@126.com and Baocan Wang; wangbaocan@xinhuamed.com.cn
}

Received 27 November 2018; Accepted 13 February 2019; Published 26 February 2019

Academic Editor: Xin-yuan Guan

Copyright (C) 2019 Ruixian Huang et al. This is an open access article distributed under the Creative Commons Attribution License, which permits unrestricted use, distribution, and reproduction in any medium, provided the original work is properly cited.

\begin{abstract}
Nonalcoholic fatty liver disease (NAFLD) is increasing in prevalence globally, but little is known about its specific molecular mechanisms. During the past decade, noncoding RNAs (ncRNAs) have been linked to NAFLD initiation and progression. They are a class of RNAs that play an important role in regulating gene expression despite not encoding proteins. This review summarizes recent research on the relationship between ncRNAs and NAFLD. We discussed the potential applicability of ncRNAs as a biomarker for early NAFLD diagnosis and assessment of disease severity. With further study, ncRNAs should prove to be valuable new targets for NAFLD treatment and benefit the development of noninvasive diagnostic methods.
\end{abstract}

\section{Introduction}

Nonalcoholic fatty liver disease (NAFLD) is the most common chronic liver disease in developed countries due to nonalcohol consumption of liver lipids. The disease exhibits a characteristic spectrum of liver damage, ranging from simple steatosis to nonalcoholic steatohepatitis (NASH), liver fibrosis, and possibly cirrhosis or even hepatocellular carcinoma (HCC) [1]. Multiple factors, including heredity, eating habits, lifestyle, and metabolic changes, all interact to induce hepatocyte injury and liver disease progression. Despite considerable research, NAFLD pathogenesis has remained unclear. However, data from the past decade have revealed that noncoding RNAs (ncRNA) may play important regulatory roles in NAFLD initiation and progression. This class of molecules, including microRNA (miRNA), long noncoding RNA (lncRNA), and circular RNA (circRNA), does not encode proteins but still influences gene expression [2].

MicroRNAs regulate posttranscriptional gene expression and are important in adipocyte differentiation, lipid metabolism, cholesterol metabolism, insulin resistance, and immune response. In vitro and in vivo models of NAFLD have shown that miRNAs affect the regulation of fatty acids
(FA) and cholesterol metabolism in the liver. In addition, they are involved in the process of regulating oxidative stress, inflammation, and apoptosis [3, 4].

Long noncoding RNAs are over 200 nucleotides. They appear to function as transcriptional regulators of gene activation or silencing through chromatin modification [5]. Posttranscriptional regulation through lncRNA involves base pairing with mRNA and using RNA-binding protein/miRNA as bait to inhibit splicing [6]. The pathophysiology of numerous diseases and cancers has been linked to lncRNA [7], but the connection with NAFLD, as well as other metabolic syndromes (e.g., obesity, insulin resistance, type 2 diabetes), remains relatively unexplored [8]. We are only beginning to understand the role of lncRNAs in NAFLD steatosis and fibrosis.

Finally, circRNA is a covalently closed-loop structure, unlike typical linear RNA that terminates with a $5^{\prime}$ cap and a $3^{\prime}$ tail. Circular RNAs are considered the main subunit of gene transcription [9]. Unsurprisingly, they are abundant, stable, and widely expressed in mammalian cells. Additionally, circRNAs exhibit cell type specificity, tissue specificity, and specific expression at different developmental stages. Recent studies have also linked circRNAs to NAFLD pathophysiology. 
In light of recent scientific developments, this review aims to summarize and elucidate the involvement of noncoding RNA in NAFLD.

\section{MiRNAs}

First described in Caenorhabditis elegans, miRNAs are endogenous, small noncoding RNAs of approximately 18-25 nucleotides [10], with a strictly controlled, multistep biosynthesis [11]. Initial miRNAs are transcribed into primary miRNA (pri-miRNA) via the action of RNA polymerase Pol II [12]. These pri-miRNAs are then processed into pre-miRNAs, hairpin structures formed via DROSHA and DICER, two ribonucleases. DROSHA cuts into the nucleus to produce pre-miRNA, which is transported from the export protein-5 (Exp5)/Ran-GTP complex to the cytoplasm. Here, DICER-TRBP-PACT cleaves pre-miRNA to produce a mature miRNA comprising a guide strand and passenger chain (or miRNA *) $[13,14]$. The guide chain is loaded onto Argonaute 2 (AGO2) and miRNA $*$ is generally considered to be degraded. Mature miRNA, AGO2, and GW182 are then loaded onto RNA-induced silencing complexes (RISC), where they bind to target mRNA [11]. This action then inhibits protein synthesis, while also adenylating and degrading the bound mRNA $[15,16]$. However, although miRNA * degradation is generally thought to be the norm, multiple studies have now demonstrated that this is not always true. Various mRNAs can target miRNA *, rather than the guide strand, suggesting that even the passenger chain plays an important regulatory role in some biological processes $[17,18]$.

Changes in miRNA expression profiles were observed at various stages of NAFLD, including simple fatty liver (SFL), NASH, and liver fibrosis to HCC. In the SFL/NASH phase, expression of miR-122, miR-34a, and miR-192 was found to be significantly elevated in both human patient and animal models [19]. At the same time, the expression of miR15b, miR125b, and miR-103 was observed to be increased in human patients [20-22], and the expression of miR-146b was decreased [23]. And in the progression and late stage of the disease, that is, the liver fibrosis/HCC stage, the expression of miR-122, miR-34a, and miR-16 was found to be elevated [2426]. In one study, normal or HFD mice either received a 16week running plan or remained sedentary. Studies have found that exercise attenuates hepatic steatosis in HFD mice, while miR-212 is overexpressed in the HFD liver and decreases after exercise [27]. This indicates that the expression of miRNA changes in the restored NAFLD, which is closely related to the process of NAFLD.

miRNA expression profiles changes have been described in human and animal NAFLD/NASH liver models [14]. Specifically, miRNA is an important regulator of liver pathophysiology and therefore influences NAFLD progression. Indeed, the role of specific miRNAs in NAFLD suggests that they can act as a potential diagnostic tool and biochemical marker. Below, we provide a summary of the main research on prominent miRNA.
2.1. $m i R-122$. The most abundant (70\%) miRNA expressed in the liver is miR-122. Transient inhibition of miR-122 expression in mice increased FA oxidation in the liver while decreasing serum cholesterol, as well as downregulating expression of cholesterol synthesis and FA synthase genes. In addition, miR-122 inhibition protects high-fat-fed mice from hepatic steatosis $[28,29]$. Similarly, inhibiting miR122 in nonhuman primates resulted in lowered cholesterol serum [30]. The above studies found that transient inhibition of miR-122 can improve liver lipid accumulation. However, germ cell knockout or liver-specific knockout miR-122 mice have lower than normal serum cholesterol, accompanied by hepatic triglyceride accumulation in juveniles. This relationship is mainly due to upregulation of miR-122 target genes associated with triglyceride biosynthesis and storage, such as Agpatl (lysophosphatidic acid acyltransferase, alpha) and Cidec (DFFA-like effector $c$ that induces cell death). In terms of diseases, miR-122 knockout mice are susceptible to NASH, fibrosis, hepatocellular carcinoma [31], timedependent steatohepatitis, and HCC; restoration of miR122 a expression reduces disease symptoms and tumorigenesis [32]. The miR-122 knockout mouse model and the transient miR-122 inhibition animal model yield inconsistent results regarding miR-122 function. More studies are necessary to clarify such a contradiction. In HepG2 cells, inhibiting miR-122 increased expression of genes involved in lipid metabolism in the liver, whereas upregulating miR-122 significantly decreased gene expression of major lipogenic enzymes [33]. Further links with lipid synthesis include the downregulation of miR-122 in free FA-induced fatty liver cells, as well as in the streptozotocin and high-fat diet- (STZHFD-) induced NASH mouse model. A major mechanism of action for miR-122 is directly targeting YY1 mRNA in the downstream (YYH-SHP) signaling cascade to regulate lipid homeostasis [34]. The above studies indicate that miR-122 is involved in the regulation of hepatic lipid metabolism in NAFLD.

In addition, circulating miR-122 is significantly upregulated in the serum of NAFLD patients in a manner strongly correlated with disease severity [35]. Recently, individuals with fatty liver were found to exhibit upregulated circulating miR-122-5p [36]. Together, existing studies demonstrate that miR-122 expression differs between hepatocytes and blood (downregulated in the former, upregulated in the latter). Thus, we suggest that circulating miR122 levels are a useful biomarker that can improve the accuracy of noninvasive NAFLD diagnosis.

2.2. $m i R-34 a$. In the liver of HFD mice, metabolic syndrome patients, and NASH patients, miR-34a is the most upregulated miRNA [33]. The upregulation of miR-34a affects postprandial response and contributes to metabolic syndrome through targeting the coreceptor beta-Klotho, which attenuates liver fibroblast growth factor 19 signaling [37]. In addition, miR-34a can target SIRT1 to trigger FA $\beta$-oxidation while reducing FA synthesis and ectopic accumulation [38]. Liver miR-34a also influences nicotinamide phosphoribosyltransferase (NAMPT), the rate-limiting enzyme in NAD+ 
biosynthesis, thereby reducing $\mathrm{NAD}(+)$ and SIRT1 levels [39]. In turn, acetylation of SIRT1 target transcriptional regulators (peroxisome proliferator-activated receptor gamma coactivator 1-alpha (PGC-1 $\alpha$ ), sterol regulatory elementbinding protein-1c (SREBP-1c), farnesoid X receptor (FXR), and nuclear factor- $\kappa \mathrm{b}(\mathrm{NF}-\kappa \mathrm{B}))$ increases, causing steatosis, inflammation, and impaired glucose tolerance.

In NAFLD and NASH patients, miR-34a overexpression inhibits SIRT1 and dephosphorylates AMP kinase, thereby lowering the phosphorylation of HMG CoA reductase and altering liver cholesterol accumulation [40]. These results indicate that miR-34a-dependent disorders to cholesterol metabolism are likely involved in NAFLD progression. In vivo and in vitro experiments revealed that miR-34a was significantly upregulated in hepatocytes from fatty liver and liver tissues of HFD-fed mice [41]. Furthermore, miR34a regulates lipid-metabolism gene expression, specifically targeting liver peroxisome proliferation-activator receptor $\alpha$ $(\operatorname{PPAR} \alpha)$ and inhibiting very low-density lipoprotein secretion, while promoting hepatic steatosis and hypolipidemic effects in a HNF4-dependent manner [42]. Recent studies have found that miR-34a concentrations decrease after transduction of the forkhead family transcription factor class $\mathrm{O} 3$ (FoxO3) in PA-induced biliary cells, suggesting that FoxO3 is a transcriptional regulator of miR-34a [43]. Furthermore, increasing miR-34a accelerates PA-induced lipid apoptosis in cholangiocarcinoma cells. In summary, miR-34a is important to the regulation of hepatic lipid metabolism and promotion of lipid apoptosis.

Unsurprisingly, given these connections, liver disease severity is associated with activation of the miR-34a/ SIRT1/p53 pathway [44], and circulating miR-34a levels is associated specifically with NAFLD severity. Liver enzyme concentrations, as well as degree of fibrosis and inflammation, are all linked to miR-34a levels, explaining why miR-34 is positively correlated with simple steatosis progression and NASH severity [25]. In conclusion, the high correlation of miR-34a with specific NAFLD pathological features [45] makes the miRNA a good candidate for a noninvasive, diagnostic biomarker of the disease.

2.3. $m i R-33 a / b$. The dual miRNAs miR-33a/b are located in introns of the sterol regulatory element-binding protein-2 (SREBP-2) and SREBP-1 genes, respectively. Because SREBP2 and SREBP-1 are involved in cholesterol and free FA metabolism, respectively [46], miR-33a/b play key roles in bile acid synthesis, FA oxidation, and cholesterol homeostasis $[47,48]$. When miR-33 is inhibited in vivo, ATP-binding cassette transporter increases in the liver, promoting cholesterol reverse-transport pathways and elevating circulating highdensity lipoprotein levels $[17,49]$. These data indicate that miR-33 is important to lipid metabolism.

While their functions are related, the two miRNAs exert different physiological effects. Elevated cholesterol inhibits miR-33a concentrations, thus increasing the expression of cholesterol 7alpha-hydroxylase (CYP7A1) and cholesterol efflux transporters. In contrast, SREBP2 and miR-33a increase intrahepatic cholesterol through downregulating cholesterol efflux transporters and bile acid synthesis [50]. Additionally,
miR-33 regulates the expression of FA-related genes [51, 52]. Specifically, miR-33a/b overexpression in vivo and in vitro reduces FA oxidation to cause triglyceride accumulation, whereas miR-33a/b targets SIRT6 [52], an important regulator of glucose metabolism. Both genes also target the AMP-dependent kinase $\alpha 1$ subunit (AMPK $\alpha 1$ ) [17, 52], which promotes $\beta$-oxidation, glucose uptake of fatty acids, and ATP synthesis. Upregulation of miR-33 also inhibits the insulin signaling pathway by targeting insulin receptor substrate-2 (IRS-2) [51, 52]. miR-33 plays a key role in lipid metabolism and insulin signaling pathways, suggesting that it could be involved in NAFLD development.

In addition to the effects of short-term anti-miR-33 treatment, a recent study found that long-term deletion of miR-33 increases triglyceride (TG) circulating levels and liver lipid deposition in HFD mice [53]. This finding underlines the need for extensive studies to observe long-term inhibition of miR-33 in nonhuman primates. Recently, it was found that liver miR33b* expression was increased in obese people and was higher in nonalcoholic steatohepatitis (NASH) within obese people [54]. This indicates that miR33b* liver expression is associated with NASH. For all this, the exact role of miR-33a/b in NAFLD and its feasibility as a new therapeutic strategy still require further investigation.

2.4. $m i R-21$. The expression of miR-21 decreased in the liver of HFD-fed mice and Hepal-6 cells treated with stearate. Furthermore, miR-21 upregulation increased the expression of FA binding protein 7 (FABP7) [55]. Serum miR-21 levels in NAFLD patients were lower than in healthy controls [56], while there was an increase in the expression of 3-hydroxy3-methylglutaryl-co-enzyme A reductase (HMGCR). Subsequent in vitro experiments then confirmed that HMGCR is a target gene of miR-21. In apparent contradiction to these findings, however, miR-21 has also been observed to increase in both obese patients and HFD mice. A potential mechanism of action involves excessive unsaturated FA, which upregulates miR-21 expression. In turn, miR-21 downregulates phosphatase and tensin homolog (PTEN) expression, leading to steatosis [57]. In mice treated with an obesogenic diet, miR21 deficiency alters the expression of multiple metabolism genes in such a way that insulin resistance, glucose tolerance, and hepatic steatosis are all aggravated [58].

The role of miR-21 in NASH has also been confirmed. Inhibiting miRNA-21 restores PPAR $\alpha$ expression in the NASH disease model, decreasing pathological symptoms in NASH-affected liver [59]. Liver, muscle, and serum biopsies of NAFLD patients revealed a major presence of miR21/PPAR $\alpha$ axis [60]. The regulatory role of miR-21 in both NAFLD and HCC appears to be through its action in the HBP1-p53-SREBP1c pathway [61].

2.5. miR-155. Widely explored in tumors, miR-155 has recently been implicated in lipogenesis that could lead to NAFLD. Specifically, miR-155 levels in the liver and peripheral blood of NAFLD patients were significantly lower than in healthy controls [62]. Increased miR-155 expression can reduce lipid metabolism-related genes SREBP1 and fatty acid 
synthase (FAS), thus lowering intracellular lipid accumulation. Downregulating miR-155 expression has the opposite effect. In vivo studies have revealed that the reduction of hepatic lipid accumulation under miR-155 upregulation occurs through inhibiting Liver X receptor (LXR) $\alpha$-dependent adipogenic signaling pathways. Fat synthesis signals and transforming growth factor (TGF) in white and brown adipose tissues can inhibit miR-155 expression to upregulation target genes and increase adipose tissue [63]. Therefore, miR155 deficiency/downregulation should elevate white adipose tissue mass, potentially leading to obesity and NAFLD.

Several studies have also begun to investigate the role of miR-155 in inflammation. Steatosis was reduced in MCDinduced miR-155-deficient mice, but this change did not attenuate associated manifestations of hepatic inflammation [64]. However, in miR-155 (-/-) mice fed with HFD for 6 months, hepatic steatosis increased [65]. Therefore, we recommend more research on $\mathrm{miR}-155$ pathophysiological mechanisms in lipid metabolism and inflammation of NAFLD and NASH patients.

2.6. $m i R-181$. Already known for its involvement in various cancers, miR-181a is overexpressed in cirrhosis and HCC [66]. In hepatocytes of insulin resistance models and in serum of diabetic patients, miR-181a is elevated, suggesting that it plays a role in NAFLD, given that insulin resistance is a major symptom. Overexpressing miR-181a has multiple effects on insulin pathways. First, it reduces SIRT1 expression in hepatocytes to induce insulin resistance [67]. Given the link between fat and insulin resistance, it is unsurprising to find that NAFLD patients, as well as HFD-treated cows and ob/ob mice, all experienced significant elevation of miR181a in blood and liver. Second, it inhibits the SIRT1-PGC$1 \alpha$ pathway and decreases insulin sensitivity, while increasing gluconeogenesis and lipid synthesis in dairy hepatocytes and HepG2 cells [68]. In addition, data from NAFLD patients and HFD-fed mice both showed that miR181b targets SIRT1 to regulate hepatic steatosis, a finding that was also confirmed in vitro [69]. Together, the available research suggests that liver miR-181 regulates insulin sensitivity and fat metabolism of NAFLD through SIRT1 inhibition.

\section{LncRNAs}

Long noncoding RNAs are either cis- or transacting in their capacity as transcriptional regulators $[70,71]$, interacting via enhancer RNAs (eRNAs) with transcription factors in the nucleus and cytoplasm [72]. Additionally, lncRNA is involved in posttranscriptional mRNA processing (splicing) $[73,74]$ and the sequestering of miRNAs away from their targets $[75,76]$. Below, we summarize recent in vivo, in vitro, and clinical trials involving lncRNAs.

3.1. Maternally Expressed Gene 3 (MEG3). Maternal expressed gene 3 (MEG3) encodes an lncRNA associated with several cancers. In CCl4-induced liver fibrosis models and human fibrotic livers, MEC3 expression was significantly reduced. Likewise, MEG3 expression was downregulated in human hepatic stellate cell line-LX-2 cells stimulated by transforming growth factor- $\beta 1$ (TGF- $\beta 1$ ) [77]. Overexpressing MEG3 in LX-2 cells appears to activate p53 and mediate cytochrome $c$ release, thus promoting caspase-3-dependent apoptosis and inhibiting TGF- $\beta 1$-induced cell proliferation. In human patients experiencing liver fibrosis and cirrhosis, liver MEG3 levels increased significantly [78]. The RNAbinding protein polypyrimidine bundle-binding protein 1 (PTBP1) interacts with MEG3, binding to the coding region of SHP, a key inhibitor of bile acid biosynthesis. This action results in rapid degradation of SHP $\mathrm{mRNA}$ and cholestasis of liver damage. The importance of MEG3 in the progression of liver fibrosis makes the gene a potential treatment target.

3.2. Alu-Mediated p21 Transcriptional Regulator (APTR). Targeted silencing RNA screening successfully identified APTR, a novel lncRNA [79] that is involved in regulating cell cycle progression and cell proliferation. Studies in two animal models of liver fibrosis ( $\mathrm{CCl} 4$ and bile duct ligation mice) have observed elevated APTR, a pattern also noted in liver fibrosis patients. Knocking down APTR can counteract $\alpha$-SMA upregulation in TGF- $\beta 1$-induced HSC in vitro, thus inhibiting HSC activation. Furthermore, APTR silencing attenuates liver fibrosis in CCl4-treated mice [80]. In the mouse liver fibrosis model, APTR accelerates cell cycle and promotes HSC proliferation through negatively regulating p21. In cirrhosis patients, serum APTR levels are higher than control, a difference also observed between patients with decompensated liver cirrhosis and those with compensated cirrhosis. Together, these data suggest that serum APTR may also be a valuable biomarker of liver fibrosis severity.

\subsection{Metastasis-Associated Lung Adenocarcinoma Transcript} 1 (MALAT1). Metastasis-associated lung adenocarcinoma transcript 1 (MALAT1) plays a key role in tumor cell proliferation, migration, and invasion. After $\mathrm{CCl} 4$ treatment, fibrotic liver tissue and activated hepatic stellate cells (HSC) both exhibited MALAT1 upregulation. Knocking down MALAT1 alleviates liver fibrosis in mice through reducing Acta2 and Collal levels. In addition, MALAT1 regulates RAS-associated C3 botulinum substrate 1 (Racl) expression via the action of miR-101b as a competitive endogenous RNA (ceRNA), affecting primary HSC proliferation, cell cycle, and activation [81].

The role of MALAT1 in hepatic steatosis and insulin resistance has also been described. For instance, MALAT1 expression increased in the livers of ob/ob mice exposed to palmitic acid HepG2 cells and type 2 diabetes mellitus animal models. Moreover, MALAT1 can promote hepatic steatosis and insulin resistance via increasing nuclear SREBP1c stability [82]. Additionally, MALAT1 regulates C-X-C motif chemokine ligand 5 expression in HepG2 cells and activates hepatic stellate cells (LX-2). Hyperglycemia and insulin both influence MALAT1 expression in HepG 2 cells, whereas only insulin affects MALAT expression in LX-2 cells [83]. These various regulatory pathways imply that MALAT1 has multiple various roles in NAFLD development. 
3.4. Plasmacytoma Variant Translocation 1 (PVT1). Plasmacytoma variant translocation 1 (PVT1) is a novel lncRNA that is upregulated in various human cancers. In fibrotic liver tissue and activated HSC, PVT1 expression is upregulated [84]. In primary HSC, PVT1 knockdown inhibits HSC activation, resulting in HSC proliferation while reducing $\alpha$ SMA and type I collagen. In vivo experiments it was also found that Pvt1 knockdown in CCl4-treated mice decreased liver collagen expression and reduced liver fibrosis. Moreover, PVT1 can function as a ceRNA for miR-152. Specifically, PVT1 competitively binds to miR-152 and inhibits PTCH1 expression through methylation, thereby activating the hedgehog pathway and promoting EMT in cirrhosis [84].

3.5. Steroid Receptor RNA Activator (SRA). When SRA is knocked out in mice, the animals experience improved glucose tolerance and reduced hepatic steatosis, thus becoming resistant to HFD-induced obesity [85]. Knocking out SRA induces expression of hepatic fatty triglyceride lipase (ATGL) under normal diets and HFD. Moreover, liver SRA and ATGL expression are inversely regulated under fasting conditions. This study found that SRA inhibits ATGL promoter activity and regulates ATGL expression primarily by inhibiting the ability of FoxO1 to promote ATGL transcription. Thus, SRA may be involved in lipid metabolism under NAFLD.

3.6. Homeobox (HOX) Transcript Antisense RNA (HOTAIR). When TGF- $\beta 1$ activated hepatic stellate cells (HSC) in the CCl4-induced mouse liver fibrosis model and in human fibrotic liver, HOTAIR expression decreased significantly [86]. In LX-2 cells, HOTAIR upregulation increased levels of alpha-smooth muscle actin ( $\alpha$-SMA), alphal(1) collagen (Collal), and fibrosis-associated genes, promoting liver fibrosis and cell proliferation. Further studies revealed that HOTAIR can function as a ceRNA for miR-148b, which promotes liver fibrosis via miR-148b regulation of DNA methyltransferase 1 (DNMT1)/MEG3/p53 pathway. Therefore, HOTAIR may be involved in the process of liver fibrosis, and of course it is necessary to further explore the role of HOTAIR in the progression of liver fibrosis in NAFLD.

3.7. Nuclear Enriched Abundant Transcript 1 (NEAT1). NEAT1 is an lncRNA that is key regulator in tumors. Expression of NEAT1 is upregulated in HCC and influences HCC cell proliferation, invasion, and migration [87]. Additionally, NEAT1, acetyl-CoA carboxylase (ACC), and fatty acid synthase (FAS) mRNA are all significantly enhanced in both in vivo and in vitro models of NAFLD [88]. Knockdown of NEAT1 reduced FCA-induced elevation of ACC and FAS in hepatocytes while significantly attenuating NAFLD performance in SD rats. Further studies found that downregulating NEAT1 levels had a therapeutic effect on NAFLD rats through the mTOR/S6K1 signaling pathway [88]. These studies all showed that NEAT1 is involved in lipid metabolism of hepatocytes.

In addition, a significant increase in NEAT1 expression was found in activated mouse hepatic stellate cells (HSC) and carbon tetrachloride- (CCl4-) induced mouse liver fibrosis models. Knockdown of NEAT1 inhibits liver fibrosis in vivo and in vitro. Overexpression of NEAT1 promotes HSC activation through increasing $\alpha$-SMA and Collal [89]. The study found that the miR-122-KLF6 axis mediates the effect of NEAT1 on HSC activation. In addition, liver samples from cirrhosis patients exhibited reduced miR-122 levels, while NEAT1 and Kruppel-like factor 6 (KLF6) increased. This outcome indicates that the NEAT1-miR-122-KLF6 axis plays a role in liver fibrosis of mice and humans. Overall, existing studies suggest that NEAT1 may play a role in NAFLD, specifically in hepatic steatosis and fibrosis.

3.8. Other IncRNAs in the Akt/SREBP-1c Pathway. Recent studies have identified a new lncRNA, lncRNA suppressor of hepatic gluconeogenesis and lipogenesis (lncSHGL). In the liver of obese mice and NAFLD patients, researchers observed decreased expression of mouse lncSHGL and its human homologous lncRNA B4GALT1-AS1 [90]. Elevating liver lncSHGL can improve hyperglycemia, insulin resistance, and steatosis in obese diabetic mice. Mechanistic studies have found that $\operatorname{lncSHGL}$ recruits heterogeneous nuclear ribonucleoprotein A1 (hnRNPA1), activates the phosphatidylinositol 3-kinase (PI3K)/Akt pathway, and inhibits the mTOR / SREBP-1C pathway, thus ameliorating hyperglycemia and steatosis in obese mice.

Increased lncARSR, FA synthesis, and oxidation-related gene expression were observed in NAFLD patients, as well as in vitro and in vivo NAFLD models [91]. Knockdown of lncARSR improved liver lipid accumulation in vivo and in vitro. Importantly, lncARSR regulates SREBP-1c expression via the PI3K/Akt pathway, which in turn regulates hepatic steatosis. These studies revealed a novel role for lncRNA in regulating hepatic steatosis via the Akt/SREBP-1c pathway.

\section{CircRNAs}

CircRNA has many conserved binding sites for miRNAs and acts as a "miRNA sponge," a term referring to competitive endogenous RNA (ceRNA) that interacts with miRNA AGO proteins to inhibit miRNA activity. The presence of miRNA sponges tends to increase miRNA target gene expression [92, 93]. This is a very important feature of circRNA functions, which can effectively bind and inhibit miRNA transcription, further affect the expression of downstream mRNA, form a circRNA-miRNA-mRNA pathway, and participate in various diseases [94]. Below, we summarized the role of circRNAs in NAFLD progression, focusing on the various circRNAs involved in disease development via the circRNA-miRNAmRNA axis.

4.1. $\operatorname{circRNA/miR-34a/PPAR\alpha }$. Both circRNA_0046367 and circRNA_0046366 are endogenous regulators of miR-34a [95, 96]. The two circRNAs block miRNA/mRNA interactions with miRNA response element (MRE) and can abolish the latter's inhibitory effect on PPAR $\alpha$. When the level of PPAR $\alpha$ is increased, its target gene carnitine palmitoyltransferase 2 (CPT2) and the acyl-CoA binding domain containing 3 (ACBD3) or the solute carrier family 27A (SLC27A) are activated, and the steatosis is reduced. These findings 
suggest that abnormal regulation of the circRNA_0046366 or circRNA_0046366/miR-34a/PPAR $\alpha$ signaling pathway may be a novel epigenetic mechanism leading to hepatic steatosis. This insight can provide a new direction for NAFLD treatment. Based on circRNA-miRNA-mRNA network analysis, decreased circRNA_021412 levels, and miR-1972 inhibition of LPIN1, the circRNA_021412/miR-1972/LPIN1 signaling cascade appears to be partially involved in regulating hepatic steatosis [97].

\section{Conclusions and Future Perspectives}

Nonalcoholic fatty liver disease is among the most prevalent chronic liver diseases globally. Its pathological features include lipid metabolism disorders, inflammation, and fibrosis. Thus, there is an urgent need for a better understanding of pathogenesis and accurate diagnostic strategies, particularly using noninvasive biomarkers. In this review, we summarized the emerging research on ncRNAs, a group of molecules that is heavily involved in NAFLD pathogenesis. ncRNA typically exhibits high cellular or tissue specificity, making them great candidates for predicting disease progression. As we learn more about the role of ncRNAs in NAFLD and other diseases, this class of molecules may become appropriate biomarkers and may also be an important reference for assessing disease severity. Among them, miRNAs are the most studied and have been implicated in lipid metabolism, insulin resistance, inflammation, fibrosis, as well as HCC development. Given their correlation with disease severity, miRNAs could be biomarkers used in early noninvasive diagnosis and assessment of NAFLD severity. Serum miRNA levels may be used as sensitive biomarkers for early detection of NAFLD. Studies have shown that the serum level of miR122 in mice with a methionine-choline deficiency (MCD) diet has increased 40-fold, far exceeding serum alanine aminotransferase (ALT) (4.8-fold) and aspartate aminotransferase (AST) (3.3-fold) [98]. It was also found that elevated levels of serum miR-122 were observed in NAFLD rats even without elevated ALT [99]. This indicates that miR-122 is more sensitive than cytokeratin (CK)-18, ALT, or AST in detecting NASH and predicting liver fibrosis in patients with NAFLD [20]. Recently, panels containing several serum miRNAs, or a combination of miRNAs and other biochemical indicators, have been shown to have a high diagnostic value for NAFLD and a higher predicted NASH potential than other biomarkers $[100,101]$. Despite this strong potential, the clinical application of miRNAs remains in its infancy. Miravirsen is a locked nucleic acid-modified DNA phosphorothioate antisense oligonucleotide that inhibits miR-122 activity and sequesters it in a stable heteroduplex. This is the first injectable miRNA-targeted drug for the treatment of HCV that has entered clinical trials, with effective and long-term reduction in viremia in treated $\mathrm{HCV}$-infected patients without serious side effects $[102,103]$. So we have reason to hypothesize that Miravirsen may has an effect on the treatment of NAFLD. Treatment of mice and African green monkeys with antagomiRs against miR-33a / b was found to be effective in increasing liver ABCA1 expression, resulting in increased high density lipoprotein (HDL) and decreased plasma levels of very high density lipoprotein (VLDL) triglycerides [17]. Therefore, oligonucleotides mimicking endogenous downregulated miRNAs in NAFLD may also be used therapeutically [104]. However, there are still many problems to be solved in the study of non-coding RNA. Contradictory results from different studies must still be resolved, and currently, miRNA detection methods have not yet been screened. Furthermore, comparative research analyzing how serum and plasma miRNAs may differ is lacking. Besides these methodological issues, further research is also necessary before we can fully address a number of major biological questions pertaining to ncRNA and NAFLD. For instance, we currently do not have a good grasp on lncRNA and circRNA function and mechanism of action in steatosis, inflammation, and fibrosis during NAFLD. We also need to investigate the relationship between lncRNA/circRNA and miRNA interactions, as well as their roles as ceRNA and miRNA sponges.

\section{Conflicts of Interest}

The authors declare that there are no conflicts of interest regarding the publication of this paper.

\section{Acknowledgments}

The authors are grateful to the National Natural Science Foundation of China and Xinhua Hospital affiliated to Shanghai Jiaotong University. This work was supported by the National Natural Science Foundation of China [No. 30900671].

\section{References}

[1] E. M. Brunt, "Pathology of nonalcoholic fatty liver disease," Nature Reviews Gastroenterology \& Hepatology, vol. 7, no. 4, pp. 195-203, 2010.

[2] M. Esteller, "Non-coding RNAs in human disease," Nature Reviews Genetics, vol. 12, no. 12, pp. 861-874, 2011.

[3] C. Fernandez-Hernando, C. M. Ramírez, L. Goedeke, and Y. Suárez, "MicroRNAs in metabolic disease," Arteriosclerosis, Thrombosis, and Vascular Biology, vol. 33, no. 2, pp. 178-185, 2013.

[4] V. Rottiers and A. M. Näär, "MicroRNAs in metabolism and metabolic disorders," Nature Reviews Molecular Cell Biology, vol. 13, no. 4, pp. 239-250, 2012.

[5] C. P. Ponting, P. L. Oliver, and W. Reik, "Evolution and functions of long noncoding RNAs," Cell, vol. 136, no. 4, pp. 629-641, 2009.

[6] J. H. Yoon, K. Abdelmohsen, and M. Gorospe, "Posttranscriptional gene regulation by long noncoding RNA," Journal of Molecular Biology, vol. 425, no. 19, pp. 3723-3730, 2013.

[7] J. K. DiStefano, "The emerging role of long noncoding rnas in human disease," Methods in Molecular Biology, vol. 1706, pp. 91110, 2018.

[8] X.-Y. Zhao and J. D. Lin, "Long noncoding RNAs: a new regulatory code in metabolic control," Trends in Biochemical Sciences, vol. 40, no. 10, pp. 586-596, 2015.

[9] J. Salzman, C. Gawad, P. L. Wang, N. Lacayo, and P. O. Brown, "Circular RNAs are the predominant transcript isoform from 
hundreds of human genes in diverse cell types," PLoS ONE, vol. 7, no. 2, Article ID e30733, 2012.

[10] R. C. Lee, R. L. Feinbaum, and V. Ambros, "The C. elegans heterochronic gene lin-4 encodes small RNAs with antisense complementarity to lin-14," Cell, vol. 75, no. 5, pp. 843-854, 1993.

[11] M. Ohtsuka, H. Ling, Y. Doki, M. Mori, and G. Calin, "MicroRNA processing and human cancer," Journal of Clinical Medicine, vol. 4, no. 8, pp. 1651-1667, 2015.

[12] H. Siomi and M. C. Siomi, "On the road to reading the RNAinterference code," Nature, vol. 457, no. 7228, pp. 396-404, 2009.

[13] E. Lund, S. Güttinger, A. Calado, J. E. Dahlberg, and U. Kutay, "Nuclear export of microRNA precursors," Science, vol. 303, no. 5654, pp. 95-98, 2004.

[14] G. Szabo and T. Csak, "Role of MicroRNAs in NAFLD/NASH," Digestive Diseases and Sciences, vol. 61, no. 5, pp. 1314-1324, 2016.

[15] M. Jinek and J. A. Doudna, "A three-dimensional view of the molecular machinery of RNA interference," Nature, vol. 457, no. 7228, pp. 405-412, 2009.

[16] J. Krol, I. Loedige, and W. Filipowicz, "The widespread regulation of microRNA biogenesis, function and decay," Nature Reviews Genetics, vol. 11, no. 9, pp. 597-610, 2010.

[17] K. J. Rayner, C. C. Esau, F. N. Hussain et al., "Inhibition of miR$33 \mathrm{a} / \mathrm{b}$ in non-human primates raises plasma HDL and lowers VLDL triglycerides," Nature, vol. 478, no. 7369, pp. 404-407, 2011.

[18] D. M. Patrick, R. L. Montgomery, X. Qi et al., "Stress-dependent cardiac remodeling occurs in the absence of microRNA-21 in mice," The Journal of Clinical Investigation, vol. 120, no. 11, pp. 3912-3916, 2010.

[19] X. L. Liu, H. X. Cao, and J. G. Fan, "MicroRNAs as biomarkers and regulators of nonalcoholic fatty liver disease," Journal of Digestive Diseases, vol. 17, no. 11, pp. 708-715, 2016.

[20] C. J. Pirola, T. F. Gianotti, G. O. Castaño et al., "Circulating microRNA signature in non-alcoholic fatty liver disease: from serum non-coding RNAs to liver histology and disease pathogenesis," Gut, vol. 64, no. 5, pp. 800-812, 2015.

[21] Y. Zhang, X. Cheng, Z. Lu et al., "Upregulation of miR-15b in NAFLD models and in the serum of patients with fatty liver disease," Diabetes Research and Clinical Practice, vol. 99, no. 3, pp. 327-334, 2013.

[22] Q. Xu, Y. Li, Y.-F. Shang, H.-L. Wang, and M.-X. Yao, "MiRNA103: Molecular link between insulin resistance and nonalcoholic fatty liver disease," World Journal of Gastroenterology, vol. 21, no. 2, pp. 511-516, 2015.

[23] M. Celikbilek, M. Baskol, S. Taheri et al., "Circulating microRNAs in patients with non-alcoholic fatty liver disease," World Journal of Hepatology, vol. 6, no. 8, pp. 613-620, 2014.

[24] M. Lu, Q. Zhang, M. Deng et al., "An analysis of human microRNA and disease associations," PLoS ONE, vol. 3, no. 10, Article ID e3420, 2008.

[25] S. Cermelli, A. Ruggieri, J. A. Marrero, G. N. Ioannou, and L. Beretta, "Circulating microRNAs in patients with chronic hepatitis C and non-alcoholic fatty liver disease," PLOS ONE, vol. 6, no. 8, Article ID e23937, 2011.

[26] H. Miyaaki, T. Ichikawa, Y. Kamo et al., "Significance of serum and hepatic microRNA-122 levels in patients with non-alcoholic fatty liver disease," Liver International, vol. 34, no. 7, pp. e302e307, 2014.

[27] J. Xiao, Y. Bei, J. Liu et al., "miR-212 downregulation contributes to the protective effect of exercise against non-alcoholic fatty liver via targeting FGF-21," Journal of Cellular and Molecular Medicine, vol. 20, no. 2, pp. 204-216, 2016.
[28] J. Krützfeldt, N. Rajewsky, R. Braich et al., "Silencing of microRNAs in vivo with 'antagomirs"' Nature, vol. 438, no. 7068, pp. 685-689, 2005.

[29] C. Esau, S. Davis, S. F. Murray et al., "miR-122 regulation of lipid metabolism revealed by in vivo antisense targeting," Cell Metabolism, vol. 3, no. 2, pp. 87-98, 2006.

[30] J. Elmén, M. Lindow, S. Schütz et al., "LNA-mediated microRNA silencing in non-human primates," Nature, vol. 452, no. 7189, pp. 896-899, 2008.

[31] S.-H. Hsu, B. Wang, J. Kota et al., "Essential metabolic, antiinflammatory, and anti-tumorigenic functions of miR-122 in liver," The Journal of Clinical Investigation, vol. 122, no. 8, pp. 2871-2883, 2012.

[32] W.-C. Tsai, S.-D. Hsu, C.-S. Hsu et al., "MicroRNA-122 plays a critical role in liver homeostasis and hepatocarcinogenesis," The Journal of Clinical Investigation, vol. 122, no. 8, pp. 2884-2897, 2012.

[33] O. Cheung, P. Puri, C. Eicken et al., "Nonalcoholic steatohepatitis is associated with altered hepatic microRNA expression," Hepatology, vol. 48, no. 6, pp. 1810-1820, 2008.

[34] G.-Y. Wu, C. Rui, J.-Q. Chen et al., "MicroRNA-122 inhibits lipid droplet formation and hepatic triglyceride accumulation via Yin Yang 1," Cellular Physiology and Biochemistry, vol. 44, no. 4, pp. 1651-1664, 2017.

[35] C. J. Pirola, T. F. Gianotti, G. O. Castaño, and S. Sookoian, "Circulating MicroRNA-122 signature in nonalcoholic fatty liver disease and cardiovascular disease: a new endocrine system in metabolic syndrome," Hepatology, vol. 57, no. 6, pp. 2545-2547, 2013.

[36] E. Raitoharju, I. Seppälä, L. Lyytikäinen et al., "Blood hsamiR-122-5p and hsa-miR-885-5p levels associate with fatty liver and related lipoprotein metabolism-The Young Finns Study," Scientific Reports, vol. 6, no. 1, article no. 38262, 2016.

[37] T. Fu, S.-E. Choi, D.-H. Kim et al., "Aberrantly elevated microRNA-34a in obesity attenuates hepatic responses to FGF19 by targeting a membrane coreceptor $\beta$-Klotho," Proceedings of the National Acadamy of Sciences of the United States of America, vol. 109, no. 40, pp. 16137-16142, 2012.

[38] A. Purushotham, T. T. Schug, Q. Xu, S. Surapureddi, X. Guo, and X. Li, "Hepatocyte-specific deletion of SIRT1 alters fatty acid metabolism and results in hepatic steatosis and inflammation," Cell Metabolism, vol. 9, no. 4, pp. 327-338, 2009.

[39] S.-E. Choi, T. Fu, S. Seok et al., "Elevated microRNA-34a in obesity reduces NAD+ levels and SIRT1 activity by directly targeting NAMPT," Aging Cell, vol. 12, no. 6, pp. 1062-1072, 2013.

[40] H.-K. Min, A. Kapoor, M. Fuchs et al., "Increased hepatic synthesis and dysregulation of cholesterol metabolism is associated with the severity of nonalcoholic fatty liver disease," Cell Metabolism, vol. 15, no. 5, pp. 665-674, 2012.

[41] J. Ding, M. Li, X. Wan et al., "Effect of miR-34a in regulating steatosis by targeting PPAR $\alpha$ expression in nonalcoholic fatty liver disease," Scientific Reports, vol. 5, no. 1, article no. 13729, 2015.

[42] Y. Xu, M. Zalzala, J. Xu, Y. Li, L. Yin, and Y. Zhang, "A metabolic stress-inducible miR-34a-HNF4 $\alpha$ pathway regulates lipid and lipoprotein metabolism," Nature Communications, vol. 6, article 7466, 2015.

[43] S. K. Natarajan, B. A. Stringham, A. M. Mohr et al., "FoxO3 increases miR-34a to cause palmitate-induced cholangiocyte lipoapoptosis," Journal of Lipid Research, vol. 58, no. 5, pp. 866875, 2017. 
[44] R. E. Castro, D. M. S. Ferreira, M. B. Afonso et al., "miR-34a/ SIRT1/p53 is suppressed by ursodeoxycholic acid in the rat liver and activated by disease severity in human non-alcoholic fatty liver disease," Journal of Hepatology, vol. 58, no. 1, pp. 119-125, 2013.

[45] V. P. Tryndyak, J. R. Latendresse, B. Montgomery et al., "Plasma microRNAs are sensitive indicators of inter-strain differences in the severity of liver injury induced in mice by a choline- and folate-deficient diet," Toxicology and Applied Pharmacology, vol. 262, no. 1, pp. 52-59, 2012.

[46] Z. Yang, T. Cappello, and L. Wang, "Emerging role of microRNAs in lipid metabolism," Acta Pharmaceutica Sinica B (APSB), vol. 5, no. 2, pp. 145-150, 2015.

[47] S. H. Najafi-Shoushtari, F. Kristo, Y. Li et al., "MicroRNA-33 and the SREBP host genes cooperate to control cholesterol homeostasis," Science, vol. 328, no. 5985, pp. 1566-1569, 2010.

[48] R. M. Allen, T. J. Marquart, C. J. Albert et al., "miR-33 controls the expression of biliary transporters, and mediates statin- and diet-induced hepatotoxicity," EMBO Molecular Medicine, vol. 4, no. 9, pp. 882-895, 2012.

[49] K. J. Rayner, Y. Suárez, A. Dávalos et al., "MiR-33 contributes to the regulation of cholesterol homeostasis," Science, vol. 328, no. 5985, pp. 1570-1573, 2010.

[50] T. Li, J. M. Francl, S. Boehme, and J. Y. L. Chiang, "Regulation of cholesterol and bile acid homeostasis by the cholesterol $7 \alpha$-hydroxylase/steroid response element-binding protein 2/microRNA-33a axis in mice," Hepatology, vol. 58, no. 3, pp. 1111-1121, 2013.

[51] I. Gerin, L.-A. Clerbaux, O. Haumont et al., "Expression of miR33 from an SREBP2 intron inhibits cholesterol export and fatty acid oxidation," The Journal of Biological Chemistry, vol. 285, no. 44, pp. 33652-33661, 2010.

[52] A. Dávalos, L. Goedeke, P. Smibert et al., "miR-33a/b contribute to the regulation of fatty acid metabolism and insulin signaling," Proceedings of the National Acadamy of Sciences of the United States of America, vol. 108, no. 22, pp. 9232-9237, 2011.

[53] L. Goedeke, A. Salerno, C. M. Ramírez et al., "Long-term therapeutic silencing of miR-33 increases circulating triglyceride levels and hepatic lipid accumulation in mice," EMBO Molecular Medicine, vol. 6, no. 9, pp. 1133-1141, 2014.

[54] T. Auguet, A. Berlanga, E. Guiu-Jurado et al., "miR33a/miR33b* and miR122 as possible contributors to hepatic lipid metabolism in obese women with nonalcoholic fatty liver disease," International Journal of Molecular Sciences, vol. 17, no. 10, 2016.

[55] J. Ahn, H. Lee, C. H. Jung, and T. Ha, "Lycopene inhibits hepatic steatosis via microRNA-21-induced downregulation of fatty acid-binding protein 7 in mice fed a high-fat diet," Molecular Nutrition \& Food Research, vol. 56, no. 11, pp. 1665-1674, 2012.

[56] C. Sun, F. Huang, X. Liu et al., "miR-21 regulates triglyceride and cholesterol metabolism in non-alcoholic fatty liver disease by targeting HMGCR," International Journal of Molecular Medicine, vol. 35, no. 3, pp. 847-853, 2015.

[57] M. Vinciguerra, A. Sgroi, C. Veyrat-Durebex, L. Rubbia-Brandt, L. H. Buhler, and M. Foti, "Unsaturated fatty acids inhibit the expression of tumor suppressor phosphatase and tensin homolog(PTEN) via microRNA-21 up-regulation in hepatocytes," Hepatology, vol. 49, no. 4, pp. 1176-1184, 2009.

[58] N. Calo, P. Ramadori, C. Sobolewski et al., "Stress-activated $\mathrm{miR}-21 / \mathrm{miR}-21 *$ in hepatocytes promotes lipid and glucose metabolic disorders associated with high-fat diet consumption," Gut, vol. 65, no. 11, pp. 1871-1881, 2016.
[59] X. Loyer, V. Paradis, C. Hénique et al., "Liver microRNA-21 is overexpressed in non-alcoholic steatohepatitis and contributes to the disease in experimental models by inhibiting PPARa expression," Gut, vol. 65, no. 11, pp. 1882-1894, 2015.

[60] P. M. Rodrigues, M. B. Afonso, A. L. Simaõ et al., "MiR-21 ablation and obeticholic acid ameliorate nonalcoholic steatohepatitis in mice," Cell Death \& Disease, vol. 8, no. 4, Article ID e2748, 2017.

[61] H. Wu, R. Ng, X. Chen, C. J. Steer, and G. Song, "MicroRNA21 is a potential link between non-alcoholic fatty liver disease and hepatocellular carcinoma via modulation of the HBP1-p53Srebplc pathway," Gut, vol. 65, no. 11, pp. 1850-1860, 2015.

[62] L. Wang, N. Zhang, Z. Wang, D.-M. Ai, Z.-Y. Cao, and H.P. Pan, "Decreased MiR-155 level in the peripheral blood of non-alcoholic fatty liver disease patients may serve as a biomarker and may influence LXR activity," Cellular Physiology and Biochemistry, vol. 39, no. 6, pp. 2239-2248, 2016.

[63] A. Virtue, C. Johnson, J. Lopez-Pastraña et al., "MicroRNA-155 deficiency leads to decreased atherosclerosis, increased white adipose tissue obesity, and non-alcoholic fatty liver disease a novel mouse model of obesity paradox," The Journal of Biological Chemistry, vol. 292, no. 4, pp. 1267-1287, 2017.

[64] T. Csak, S. Bala, D. Lippai et al., "MicroRNA-155 deficiency attenuates liver steatosis and fibrosis without reducing inflammation in a mouse model of steatohepatitis," PLOS ONE, vol. 10, no. 6, Article ID e0129251, 2015.

[65] A. M. Miller, D. S. Gilchrist, J. Nijjar et al., "MiR-155 has a protective role in the development of non-alcoholic hepatosteatosis in mice," PLoS ONE, vol. 8, no. 8, Article ID e72324, 2013.

[66] J. Brockhausen, S. S. Tay, C. A. Grzelak et al., "miR-181a mediates TGF- $\beta$-induced hepatocyte EMT and is dysregulated in cirrhosis and hepatocellular cancer," Liver International, vol. 35, no. 1, pp. 240-253, 2015.

[67] B. Zhou, C. Li, W. Qi et al., "Downregulation of miR-181a upregulates sirtuin-1 (SIRT1) and improves hepatic insulin sensitivity," Diabetologia, vol. 55, no. 7, pp. 2032-2043, 2012.

[68] X. Du, Y. Yang, C. Xu et al., "Upregulation of miR-181a impairs hepatic glucose and lipid homeostasis," Oncotarget , vol. 8, no. 53, pp. 91362-91378, 2017.

[69] Y. Wang, K. Zhu, W. Yu et al., "MiR-181b regulates steatosis in nonalcoholic fatty liver disease via targeting SIRT1," Biochemical and Biophysical Research Communications, vol. 493, no. 1, pp. 227-232, 2017.

[70] J. T. Lee and M. S. Bartolomei, "X-inactivation, imprinting, and long noncoding RNAs in health and disease," Cell, vol. 152, no. 6, pp. 1308-1323, 2013.

[71] J. L. Rinn, M. Kertesz, J. K. Wang et al., "Functional demarcation of active and silent chromatin domains in human HOX loci by noncoding RNAs," Cell, vol. 129, no. 7, pp. 1311-1323, 2007.

[72] N. E. Iiott, J. A. Heward, B. Roux et al., "Long non-coding RNAs and enhancer RNAs regulate the lipopolysaccharideinduced inflammatory response in human monocytes," Nature Communications, vol. 5, article no. 3979, 2014.

[73] M. Beltran, I. Puig, C. Pena et al., "A natural antisense transcript regulates Zeb2/Sip1 gene expression during Snaill-induced epithelial-mesenchymal transition," Genes \& Development, vol. 22, pp. 756-769, 2008.

[74] V. Tripathi, J. D. Ellis, Z. Shen et al., "The nuclear-retained noncoding RNA MALAT1 regulates alternative splicing by modulating SR splicing factor phosphorylation," Molecular Cell, vol. 39, no. 6, pp. 925-938, 2010. 
[75] C. Gong and L. E. Maquat, "Maquat, lncRNAs transactivate STAU1-mediated mRNA decay by duplexing with $3^{\prime}$ UTRs via Alu elements," Nature, vol. 470, no. 7333, pp. 284-288, 2011.

[76] J. H. Yoon, K. Abdelmohsen, S. Srikantan et al., "LincRNA-p21 suppresses target mRNA translation," Molecular Cell, vol. 47, pp. 648-655, 2012.

[77] Y. He, Y.-T. Wu, C. Huang et al., "Inhibitory effects of long noncoding RNA MEG3 on hepatic stellate cells activation and liver fibrogenesis," Biochimica et Biophysica Acta (BBA) Molecular Basis of Disease, vol. 1842, no. 11, pp. 2204-2215, 2014.

[78] L. Zhang, Z. Yang, J. Trottier, O. Barbier, and L. Wang, "Long noncoding RNA MEG3 induces cholestatic liver injury by interaction with PTBP1 to facilitate shp mRNA decay," Hepatology, vol. 65 , no. 2, pp. 604-615, 2017.

[79] M. Negishi, S. P. Wongpalee, S. Sarkar et al., "A new lncRNA, APTR, associates with and represses the CDKN1A/p21 promoter by recruiting polycomb proteins," PLoS ONE, vol. 9, no. 4, 2014.

[80] F. Yu, J. Zheng, Y. Mao et al., "Long non-coding RNA APTR promotes the activation of hepatic stellate cells and the progression of liver fibrosis," Biochemical and Biophysical Research Communications, vol. 463, no. 4, pp. 679-685, 2015.

[81] F. Yu, Z. Lu, J. Cai et al., "MALAT1 functions as a competing endogenous RNA to mediate Racl expression by sequestering miR-101b in liver fibrosis," Cell Cycle, vol. 14, no. 24, pp. 38853896, 2015.

[82] C. Yan, J. Chen, and N. Chen, "Long noncoding RNA MALAT1 promotes hepatic steatosis and insulin resistance by increasing nuclear SREBP-1c protein stability," Scientific Reports, vol. 6, 2016.

[83] F. Leti, C. Legendre, C. D. Still et al., "Altered expression of MALAT1 lncRNA in nonalcoholic steatohepatitis fibrosis regulates CXCL5 in hepatic stellate cells," Translational Research, vol. 190, pp. 25.e21-39.e21, 2017.

[84] J. Zheng, F. Yu, P. Dong et al., "Long non-coding RNA PVT1 activates hepatic stellate cells through competitively binding microRNA-152," Oncotarget , vol. 7, no. 39, pp. 62886-62897, 2016.

[85] G. Chen, D. Yu, X. Nian et al., "LncRNA SRA promotes hepatic steatosis through repressing the expression of adipose triglyceride lipase (ATGL)," Scientific Reports, vol. 6, article no. 35531, 2016.

[86] E. Bian, Y. Wang, Y. Yang et al., "Hotair facilitates hepatic stellate cells activation and fibrogenesis in the liver," Biochimica et Biophysica Acta (BBA) - Molecular Basis of Disease, vol. 1863, no. 3, pp. 674-686, 2017.

[87] Y. Mang, L. Li, J. Ran et al., "Long noncoding RNA NEAT1 promotes cell proliferation and invasion by regulating hnRNP A2 expression in hepatocellular carcinoma cells," OncoTargets and Therapy, vol. 10, pp. 1003-1016, 2017.

[88] X. Wang, "Down-regulation of IncRNA-NEAT1 alleviated the non-alcoholic fatty liver disease via mTOR/S6K1 signaling pathway," Journal of Cellular Biochemistry, vol. 119, no. 2, pp. 1567-1574, 2018.

[89] F. Yu, Z. Jiang, B. Chen, P. Dong, and J. Zheng, "NEAT1 accelerates the progression of liver fibrosis via regulation of microRNA-122 and Kruppel-like factor 6," Journal of Molecular Medicine, vol. 95, no. 11, pp. 1191-1202, 2017.

[90] J. Wang, W. Yang, Z. Chen et al., "Long noncoding RNA lncSHGL recruits hnRNPA1 to suppress hepatic gluconeogenesis and lipogenesis," Diabetes, vol. 67, no. 4, pp. 581-593, 2018.
[91] M. Zhang, X. Chi, N. Qu, and C. Wang, "Long noncoding RNA lncARSR promotes hepatic lipogenesis via Akt/SREBP-1c pathway and contributes to the pathogenesis of nonalcoholic steatohepatitis," Biochemical and Biophysical Research Communications, vol. 499, no. 1, pp. 66-70, 2018.

[92] S. Memczak, M. Jens, A. Elefsinioti et al., "Circular RNAs are a large class of animal RNAs with regulatory potency," Nature, vol. 495, no. 7441, pp. 333-338, 2013.

[93] T. B. Hansen, T. I. Jensen, B. H. Clausen et al., "Natural RNA circles function as efficient microRNA sponges," Nature, vol. 495, no. 7441, pp. 384-388, 2013.

[94] J. E. Wilusz and P. A. Sharp, "A circuitous route to noncoding RNA," Science, vol. 340, no. 6131, pp. 440-441, 2013.

[95] X.-Y. Guo, J.-N. Chen, F. Sun, Y.-Q. Wang, Q. Pan, and J.-G. Fan, "CircRNA-0046367 prevents hepatoxicity of lipid peroxidation: an inhibitory role against hepatic steatosis," Oxidative Medicine and Cellular Longevity, vol. 2017, Article ID 3960197, 16 pages, 2017.

[96] X.-Y. Guo, F. Sun, J.-N. Chen, Y.-Q. Wang, Q. Pan, and J.-G. Fan, "CircRNA-0046366 inhibits hepatocellular steatosis by normalization of PPAR signaling," World Journal of Gastroenterology, vol. 24, no. 3, pp. 323-337, 2018.

[97] X.-Y. Guo, C.-X. He, Y.-Q. Wang et al., "Circular RNA profiling and bioinformatic modeling identify its regulatory role in hepatic steatosis," BioMed Research International, vol. 2017, Article ID 5936171, 13 pages, 2017.

[98] J. D. Clarke, T. Sharapova, A. D. Lake, E. Blomme, J. Maher, and N. J. Cherrington, "Circulating microRNA 122 in the methionine and choline-deficient mouse model of non-alcoholic steatohepatitis," Journal of Applied Toxicology, vol. 34, no. 6, pp. 726-732, 2014.

[99] H. Yamada, K. Ohashi, K. Suzuki et al., "Longitudinal study of circulating miR-122 in a rat model of non-alcoholic fatty liver disease," Clinica Chimica Acta, vol. 446, pp. 267-271, 2015.

[100] P. P. Becker, M. Rau, J. Schmitt et al., "Performance of serum microRNAs-122,-192 and-21 as biomarkers in patients with nonAlcoholic steatohepatitis," PLoS ONE, vol. 10, no. 11, Article ID e0142661, 2015.

[101] Y. Tan, G. Ge, T. Pan, D. Wen, and J. Gan, "A pilot study of serum microRNAs panel as potential biomarkers for diagnosis of nonalcoholic fatty liver disease," PLoS ONE, vol. 9, no. 8, Article ID e105192, 2014.

[102] H. L. A. Janssen, H. W. Reesink, E. J. Lawitz et al., "Treatment of HCV infection by targeting microRNA," The New England Journal of Medicine, vol. 368, no. 18, pp. 1685-1694, 2013.

[103] J. Hu, Y. Xu, J. Hao, S. Wang, C. Li, and S. Meng, "MiR-122 in hepatic function and liver diseases," Protein \& Cell, vol. 3, pp. 364-371, 2012.

[104] G. Szabo, P. Sarnow, and S. Bala, "MicroRNA silencing and the development of novel therapies for liver disease," Journal of Hepatology, vol. 57, no. 2, pp. 462-466, 2012. 


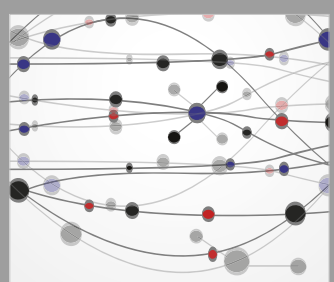

The Scientific World Journal
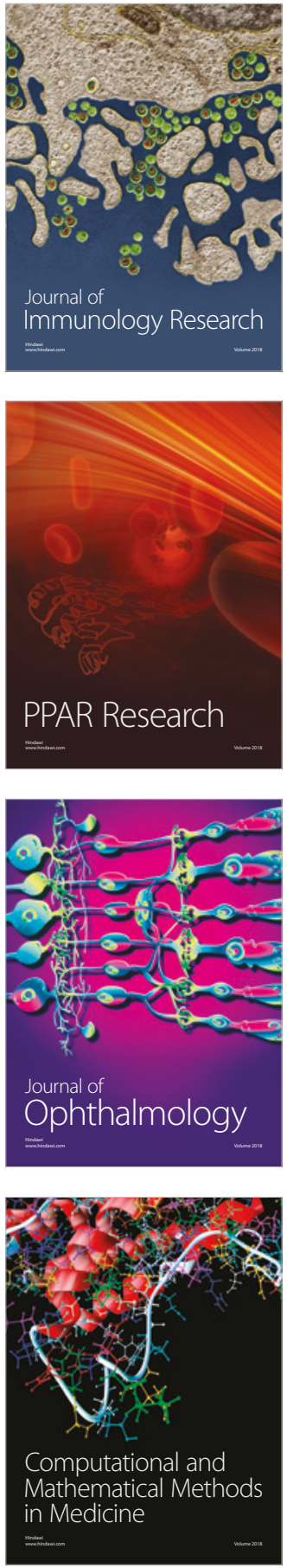

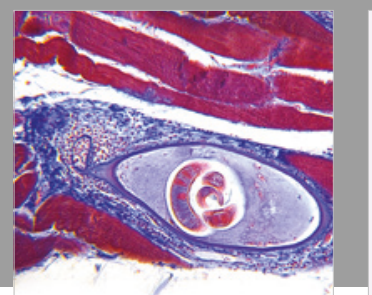

Gastroenterology Research and Practice

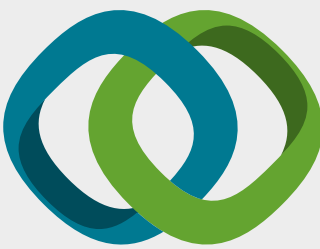

\section{Hindawi}

Submit your manuscripts at

www.hindawi.com
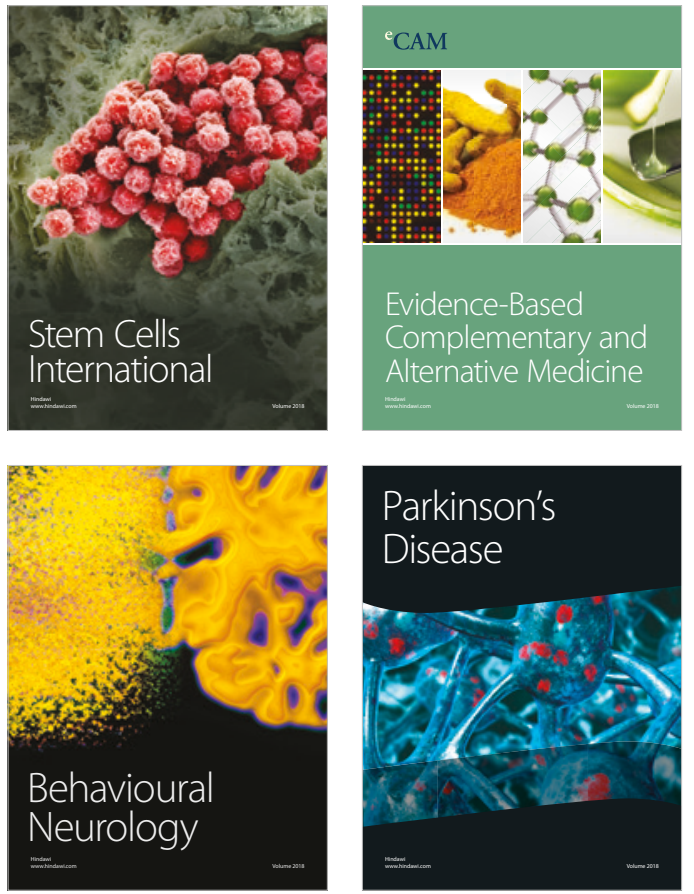

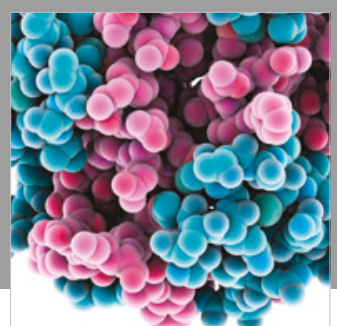

ournal of

Diabetes Research

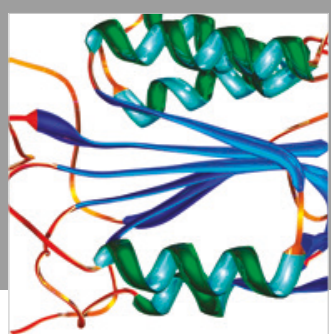

Disease Markers
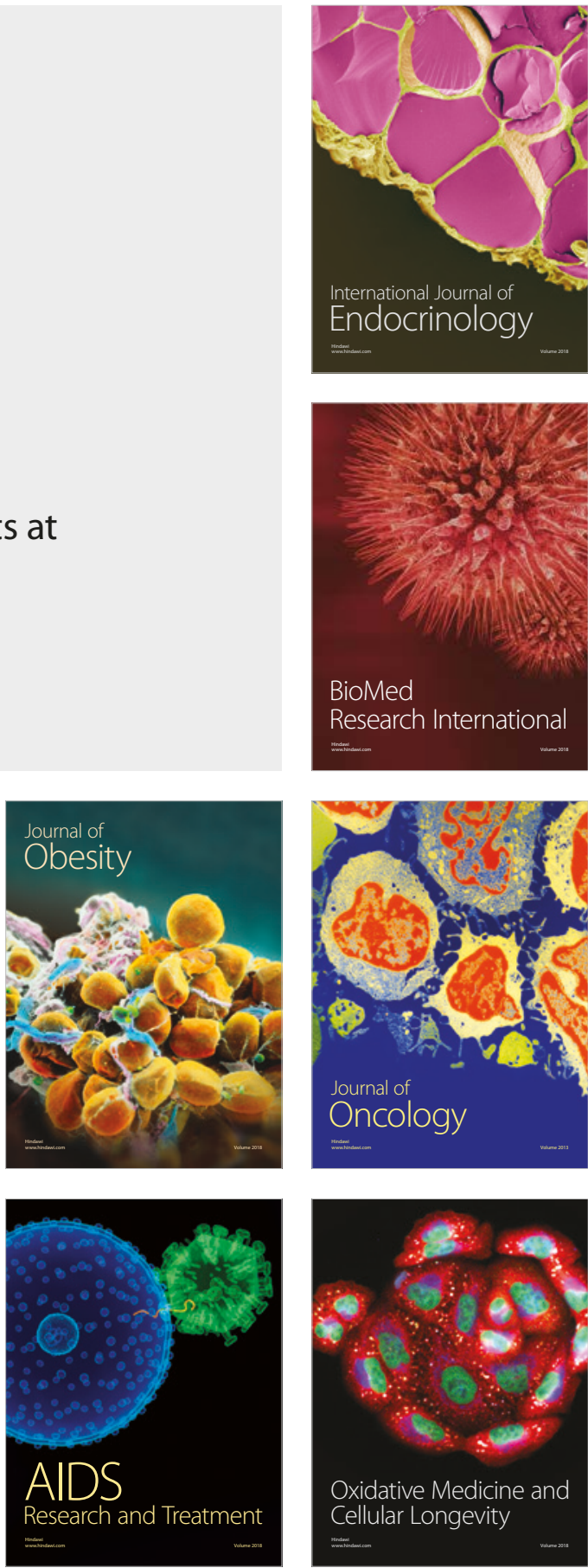